\title{
Tutoría entre iguales como estrategia para la formación del profesorado
}

Carolina Villagra-Bravo cvillagra@uct.cl

Universidad Católica de Temuco, Chile https://orcid.org/0000-0002-5428-2555

VAnessa Valdebenito-Zambrano vvaldebenito@uct.cl Universidad CATólica de Temuco, Chile https://orcid.org/0000-0002-5279-563X

\begin{abstract}
Resumen
La investigación es un estudio de caso con metodología mixta que involucra un análisis cuantitativo con grupo de comparación respecto al nivel de logro y un análisis cualitativo de la valoración que tutores y tutorados dan a la tutoría. Los resultados evidencian una mejora en las dimensiones evaluadas - conocimiento y habilidades - para el grupo de intervención, y no así para el grupo control. La tutoría entre iguales constituye una estrategia que favorece el aprendizaje en el contexto universitario, pues brinda la posibilidad de construir conocimiento desde ambos roles asumidos por los estudiantes (tutores y tutorados).
\end{abstract}

\section{Palabras clave}

Método de aprendizaje; relaciones entre pares; enseñanza superior; formación de profesores

\begin{abstract}
This research is a case study with combined methodologies involving a quantitative analysis with a group comparison regarding their level of achievement and a qualitative analysis of the assessment by both peers taking part in a peer coaching. The results show an improvement in the assessed dimensions -knowledge and skills- in the intervention group but not in the control group. Peer coaching constitutes a strategy favoring the learning in the college context as it provides an opportunity to build knowledge from both roles performed by the peers (the coach and the student).
\end{abstract}

\section{Keywords}

Learning method; peer relationship; higher education; teacher education

Para citar este artículo / To cite this article

Villagra-Bravo, C. \& Valdebenito-Zambrano, V. (2019). Tutoría entre iguales como estrategia para la formación del profesorado. magis, Revista Internacional de Investigación en Educación, 12 (24), 161-176. doi: 10.11144/Javeriana.m12-24.tief 


\section{Introducción}

La actual sociedad del conocimiento demanda a sus miembros metas cada vez más exigentes, provenientes — como señala Ángel Parrilla Latas (2002) - de la globalización, modernización, conocimiento científico y tecnológico, cambios y contingencias políticas, además de las demandas del mercado. En consecuencia, varios sistemas educativos europeos y latinoamericanos han definido programas por competencias para la formación profesional.

En el contexto chileno, y en lo que se refiere a educación superior, varias universidades están inmersas en una corriente de renovación, que involucra la enseñanza por competencias como elemento central del currículo, que bien resume el proyecto Tuning Latinoamérica (Beneitone, Esquetini, González, Maletá, Siufi \& Wagenaar, 2007). La formación por competencias responde a las exigencias del contexto globalizado y favorecen las bases que permiten la adaptación a los cambios en el saber (aprender a hacer) y una proyección fuera del contexto educativo formal (Garagorri-Yarza, 2007; Pérez-Gómez, 2007; Zabala \& Arnau, 2007).

Como destaca el proyecto Tuning para Latinoamérica (Beneitone, Esquetini, González, Maletá, Siufi \& Wagenaar, 2007), una de las necesidades de las universidades latinoamericanas es la transformación del paradigma educativo, desde un proceso centrado exclusivamente en la enseñanza, en otro centrado en el estudiante y su proceso de aprendizaje. Bajo este paradigma, el profesorado ofrece a sus estudiantes mayores y diversas oportunidades para la construcción de su conocimiento y propicia una gestión metacognitiva del proceso de aprendizaje. Esta transformación implica plantearse la relación e interacción entre los miembros del aula y no solo la relación profesor-estudiante como generadora de conocimiento de la educación tradicional (Duran \& Vidal-Iglesias, 2004).

Por su parte, la Organización de las Naciones Unidas para la Educación, la Ciencia y la Cultura, UNESCO (Delors, 1996), y la Organización para la Cooperación y el Desarrollo Económicos, OCDE (2005), seleccionan algunas competencias como claves o básicas para el desarrollo y aprendizaje; la interacción en grupos diversos se destaca como fundamental para el desarrollo del individuo. En este estudio relacionamos esta competencia con el aprendizaje entre iguales. Entendemos que estructurar las interacciones de los individuos a través de métodos de aprendizaje cooperativo - como se presenta en esta investigación - brinda oportunidades de aprendizaje a los estudiantes a través del diálogo, la reflexión y la negociación de significados.

En este estudio, tomamos uno de los métodos de aprendizaje cooperativo más implementados y estudiados empíricamente (Topping, Duran \& Van Keer, 2015), denominado tutoría entre iguales - peer tutoring(Topping, 1996). La tutoría entre iguales es definida por David Duran y Vinyet Vidal-Iglesias (2004) como la creación de parejas de personas que asumen una relación asimétrica, derivada de la adopción de un rol de tutor - maestro — y/o tutorado - aprendiz — , que trabajan por un objetivo común, conocido y compartido en un marco de interacción planificado y regulado por el profesor. En este caso, para la formación de profesores, se ha implementado específicamente como una estrategia didáctica que permite favorecer la construcción de conocimiento, que fortalece el aprendizaje y la enseñanza por competencias. Esta estrategia permite además sacar provecho pedagógico de las diferencias y favorecer la construcción de nuevos aprendizajes (Monereo-Font, 2001). 
Tutoria entre iguales en la Universidad

La tutoría entre iguales se entiende como un método de aprendizaje cooperativo, que considera la creación de grupos pequeños de estudiantes que trabajan de manera colaborativa y alcanzan objetivos comunes (Gillies, 2007; Good \& Brophy, 2000). En este sentido, la tutoría entre iguales es uno de los métodos de aprendizaje cooperativo y una de las estrategias didácticas más potentes y validadas en diversos contextos de práctica para la educación de calidad (González-Fernández, García-Ruiz \& Ramírez-García, 2015; Topping, 2000). Keith J. Topping (1988) distingue dos tipos de tutoría: cross age tutoring, tutorías entre estudiantes de diferente edad y nivel (que asumen roles fijos), y same age tutoring, en que los participantes tienen edades y niveles similares (que pueden asumir roles fijos o intercambiables).

Los estudios en esta área muestran resultados favorables en sus intervenciones, tanto en tutoría fija (Fuchs, Fuchs, Hamlett, Phillips, Karns \& Dutka, 1997; Valdebenito \& Duran, 2015), recíproca (Blanch, Duran, Dekhinet \& Topping, 2010; Mastropieri, Scruggs, Spencer \& Fontana, 2003) y para ambas (Duran \& Monereo, 2005). Asimismo, han reportado los beneficios de esta estrategia didáctica en diferentes ámbitos y niveles escolares, como atención a la diversidad (Mastropieri, Scruggs, Norland, Berkeley, McDuffie, Halloran Tornquist \& Connors, 2006; Mortweet, Utley, Walker, Dawson, Delquadri, Reddy, Greenwood, Hamilton \& Ledford, 1999; Robinson, Schofield \& Steers-Wentzel, 2005; Ryan, Reid \& Epstein, 2004); ciencias (Thurston, Van de Keere, Topping, Kosack, Gatt, Marchal, Mestdagh, Schmeinck, Sidor \& Donnert, 2007); competencia lectora (Blanch, Duran, Valdebenito \& Flores, 2013; Duran \& Utset, 2014; Moliner-Miravet, FloresColl \& Duran-Gisbert, 2011); escritura (Blanch-Gelabert, Corcelles-Seuba, Duran-Gisbert, Dekhinet \& Topping, 2014); y autoconcepto (Flores \& Duran, 2016), entre otros. Además, evidencian beneficios transversales de la tutoría como ampliación de expectativas, habilidades de pensamiento, relaciones sociales, resolución de conflictos y negociación de significados, entre otros.

En el ámbito universitario, la tutoría entre iguales se ha desarrollado en el contexto anglosajón y europeo principalmente, con incipientes investigaciones exploratorias en Latinoamérica, que dan cuenta de satisfactorios resultados (Alzate Medina \& Peña Borrero, 2010; Duran \& Flores, 2015; Duran, Flores, Mosca \& Santiviago, 2014; Duran \& Sánchez-Chacón, 2012; Mosca \& Santiviago, 2012). En este contexto, la tutoría entre iguales ha permitido explorar los itinerarios dialógicos establecidos entre estudiantes, la regulación metacognitiva, el autoconcepto académico, las competencias socioprofesionales (Álvarez-Pérez \& González-Afonso, 2005; Duran \& Huerta-Córdova, 2008) y las ganancias académicas (De Backer, Van Keer \& Valcke, 2016; González-Fernández, García-Ruiz \& Ramírez-García, 2015; Moliner-Miravet, Sales-Ciges \& Moliner-García, 2014).

Por otra parte, Claudio G. Cortese (2005) destaca que en la tutoría entre iguales ambos participantes se benefician en coherencia con el paradigma aprender enseñando (learning by teaching). En estos casos, el mayor incremento en el aprendizaje es atribuido por el enseñante o tutor al desafío cognitivo que significa enseñar a otros. Según David Duran (2014), ello se debe a que dicha actividad permite cuestionar el pensamiento tradicional de enseñanza, y centrarlo en el aprendizaje; en este caso, no solo del tutorado, sino también de quien asume el rol de tutor.

Rod D. Roscoe y Michelene T. H. Chi (2007), en una revisión exhaustiva, evidencian mayores ganancias cognitivas para el tutor en diferentes tipos de tutoría, niveles, áreas curriculares, orígenes culturales y étnicos, 
que explican a qué se debería esta situación. Estos autores plantean que en primer lugar se desarrolla la actividad de explicación, que considera comprometerse en la construcción reflexiva del conocimiento, a través de la generación de explicaciones coherentes, internamente consistentes, reorganización mental, y evaluación consciente del proceso y de sus conocimientos. Posteriormente, se desarrolla la interrogación para introducir nuevos temas, guiar y evaluar el proceso, tal como lo hace un profesor, y estar preparado para dar respuestas a las preguntas planteadas por su compañero o aprendiz.

Consideramos relevante la tutoría entre iguales como estrategia didáctica para la formación de profesores, con el propósito de promover aprendizajes más integrales y brindar oportunidades a los futuros maestros para desenvolverse de forma anticipada en otros contextos de enseñanza. Por otra parte, la tutoría entre iguales como estrategia para la formación favorece el desarrollo de prácticas más inclusivas en el nivel universitario que responden a los diversos desafíos en esta etapa, no solo en términos de aprobación-reprobación o retención-deserción, sino en términos más cualitativos, que consideren los procesos de incorporación desde una perspectiva más personal y social.

Desde hace algunos años, mejorar la formación de profesores constituyó una de las Metas educativas 2021, de la Organización de Estados Iberoamericanos para la Educación, la Ciencia y la Cultura (OEI, 2010). Tradicionalmente, los modelos y enfoques de la formación de profesores se basan en paradigmas conservadores de los procesos pedagógicos de enseñanza y aprendizaje, sin responder a las necesidades de la sociedad del conocimiento (Vezub, 2007).

En las aulas universitarias se deben considerar actividades de aprendizaje que permitan al futuro docente vivir experiencias que queremos que replique luego cuando enseñe (Ezquerra-Martínez, De Juanas-Oliva \& Martín del Pozo, 2015). De este modo, democratizar el aula a través de estrategias didácticas como la tutoría entre iguales, permitiría a los futuros profesores enseñar y enseñar a aprender desde un enfoque constructivista, participativo, interaccionista y situado.

Proyecto universitario de tutoría entre iguales

Esta investigación da cuenta de un proyecto piloto, puesto en marcha en la asignatura de Psicología del aprendizaje y del desarrollo, de un programa de formación inicial de profesores de Educación Básica de la Universidad Católica de Temuco, Chile. Es importante mencionar que, desde 2009, la universidad ha implementado un nuevo modelo educativo, que considera la formación profesional basada en competencias como uno de sus ejes. Se distinguen dos tipos de competencias: específicas, propias de cada área de formación disciplinaria, y genéricas, relacionadas con el desarrollo integral de los futuros profesionales en su dimensión personal e interpersonal. Estas competencias genéricas constituyen el sello formativo común de la institución (Universidad Católica de Temuco, 2007).

En relación con la asignatura foco del proyecto implementado, históricamente alcanza más de un 35\% de reprobación. Esta situación obstaculiza el avance curricular de los estudiantes y afecta los índices de eficiencia interna del programa de formación, en particular en los porcentajes de aprobación y titulación oportuna.

1 Educación primaria. 
Los objetivos del estudio son:

a. Medir el impacto de un programa de tutoría entre iguales en el rendimiento de estudiantes universitarios de primer año en una asignatura de Psicología del aprendizaje en contraste con un grupo de comparación de referencia.

b. Describir las valoraciones de tutores y tutorados en relación con sus aprendizajes y en función de las ayudas entregadas y recibidas en el programa de tutoría entre iguales.

\section{Metodología}

La investigación que se presenta es un estudio de caso y se enmarca en la investigación educativa para aportar conocimiento, y comprender y mejorar los procesos educativos de un caso en particular (Sabariego \& Bisquerra, 2004; Stake, 2005).

La metodología es mixta, pues combina un estudio cuantitativo de carácter descriptivo que considera el nivel de logro de los estudiantes al finalizar el semestre académico. Además, por medio de un análisis cualitativo, se indaga la valoración del programa y los aprendizajes construidos desde la mirada de los estudiantes. Esta complementariedad facilita una aproximación y comprensión más completa sobre el objeto de estudio.

Los participantes en esta investigación se organizan en dos grupos, uno de intervención y otro de control. El grupo de intervención estaba conformado por 22 estudiantes de primer año de la carrera de Pedagogía en Educación Básica, los cuales asistieron de manera regular a la asignatura de Psicología del aprendizaje y del desarrollo, que se cursa al inicio del programa formativo de pedagogía, específicamente en el primer semestre. Estos alumnos fueron invitados a participar como tutorados del programa tomando en cuenta las calificaciones iniciales obtenidas en la asignatura de psicología y los resultados de las pruebas de diagnóstico aplicadas durante la primera semana de ingreso a la universidad. Las áreas evaluadas y en las que los estudiantes presentaron más bajos resultados se refieren a comprensión de textos, técnicas de estudio y habilidades sociales.

Además, participan 12 estudiantes destacados de cursos superiores de la misma titulación que toman el rol de tutores, junto con una docente con formación y experiencia en el área de aprendizaje cooperativo y tutoría entre iguales, que orienta el proceso de acompañamiento entre pares.

El grupo de comparación corresponde a otro curso de Psicología del aprendizaje y del desarrollo compuesto también por 22 estudiantes, que pertenecen a otros programas formativos de pedagogía de la misma Facultad de Educación y que poseen características similares al grupo de intervención (calificaciones iniciales deficientes, bajos resultados en pruebas diagnósticas sobre comprensión de textos, habilidades y técnicas de estudio y habilidades sociales). Este grupo no participa de la experiencia de tutoría entre iguales, por tanto, está expuesto a estrategias didácticas comunes.

\section{Descripción del programa de tutoría entre iguales y procedimiento}

de la investigación

En la etapa de implementación de este programa se identifican 3 fases:

Fase 1. Postulación y formación de tutores: en el período de un mes se convocó a estudiantes de cursos superiores de pedagogía a postular y participar como tutores en un programa de tutoría entre iguales. La selección se basó en calificaciones y resultados de un cuestionario que mide habilidades sociales en cinco dimensiones: habilidades empáticas y solidarias; 
conversacionales; de autoafirmación; de colaboración y de resolución de conflictos (Tapia-Gutiérrez, 2012).

Después de la selección, se desarrolló el proceso de formación a cargo de la profesora experta, que lideró las tres sesiones estructuradas en las orientaciones de la literatura en tutoría entre iguales (Duran, Blanch, Corcelles, Flores, Oller, Utset \& Valdebenito, 2011; Topping, Duran \& Van Keer, 2015). Las sesiones de formación involucraron: a) objetivos de la propuesta, conocimiento de las bases de la tutoría entre iguales y comprensión del aprendizaje de ambos roles - tutor y tutorado-; b) objetivos del programa de tutoría, contenidos a trabajar y estructura de las actividades; c) familiarización con los materiales de trabajo, presentación y conocimiento de las parejas, compromisos y normas.

Fase 2. Tutoría entre iguales y evaluación de seguimiento: considera las acciones de tutorías entre pares de la carrera de Pedagogía en Educación Básica con Mención, en las cuales un tutor acompaña a dos estudiantes que cursan la asignatura de primer año Psicología del aprendizaje y del desarrollo. Durante el semestre académico en el que se desarrolla el curso, los estudiantes de pedagogía se reúnen dos veces a la semana según horarios disponibles de su tiempo autónomo, por un período promedio de dos horas semanales. En total se realizaron 22 sesiones de trabajo presencial entre tutor y tutorados, las cuales se enfocaron en actividades vinculadas a objetivos y contenidos de la asignatura con énfasis en el ciclo vital de infancia, adolescencia y teorías psicoeducativas del aprendizaje, a través de negociación de significados, colaboración y metacognición del proceso de aprendizaje.

Además, los tutores se reunían cada dos semanas con la profesora a cargo de la asignatura y el programa para recibir orientaciones y retroalimentación sobre los avances en el proceso de aprendizaje de sus tutorados, para despejar posibles dudas y plantear posibles formas para facilitar la construcción del conocimiento de sus pares. Adicionalmente, en estos espacios se realiza seguimiento de la estrategia y se revisa el cumplimiento de los compromisos asumidos por tutores y tutorados al inicio del programa.

Fase 3. Evaluación final: corresponde al período posterior al desarrollo de las tutorías. En esta fase, se realizaron las evaluaciones finales de la asignatura y se recogió la opinión de tutores y tutorados que participaron del programa.

Instrumentos y técnicas de recogida de datos

Para recoger los datos, se utilizaron las siguientes técnicas e instrumentos:

a. Nivel de logro en la asignatura: al finalizar el semestre académico, se aplicó una prueba escrita a los estudiantes del grupo de intervención y de control, para evaluar el logro de los objetivos de la asignatura de Psicología del aprendizaje y del desarrollo. El instrumento de evaluación contempla dos dimensiones, una basada en el conocimiento construido de la asignatura y la otra que contempla las habilidades para aplicar ese conocimiento en casos o situaciones educativas.

b. Grupos focales a tutores y tutorados: se realizaron dos grupos focales con 10 participantes en cada caso, diferenciados en el rol que asumieron en el programa. Las temáticas abordadas se relacionaron con indagar la satisfacción con el rol asumido, dificultades, búsqueda de soluciones, aprendizajes y valoración general de la experiencia. 


\section{Análisis de resultados}

Para indagar sobre los resultados cuantitativos, se aplicó un análisis no paramétrico a través de la prueba $W$ de Wilcoxon, porque de acuerdo al tamaño de las muestras no es posible determinar una distribución normal. Los resultados de la prueba escrita considerados para el análisis se definieron con un porcentaje de logro en una escala de 0 a $100 \%$.

En el caso del análisis cualitativo se analizaron los discursos manifiestos por los participantes del programa. Se utilizó un software especializado para la codificación de datos y el levantamiento de categorías que dan cuenta de las valoraciones que hacen tutores y tutorados, respecto de su experiencia con la tutoría entre iguales.

\section{Resultados}

Los resultados se organizan en función de los objetivos de estudio.

a. Diferencia entre el nivel de logro de estudiantes universitarios que participan de un programa de tutoría entre iguales en contraste con un grupo de comparación

Como se observa en la tabla 1, el grado de significancia es menor que .05, por tanto, se rechaza la hipótesis nula y se evidencia que el grupo de intervención obtiene mayor nivel de logro que el grupo de control y que esta diferencia es estadísticamente significativa: $z=-2,188 ; p=.029$.

Tabla 1

Prueba no paramétrica

\begin{tabular}{l|c}
\hline & $\begin{array}{c}\text { Porcentaje de logro } \\
\text { en la prueba }\end{array}$ \\
\hline U de Mann-Whitney & 149,000 \\
\hline W de Wilcoxon & 402,000 \\
\hline$Z$ & $-2,188$ \\
\hline Significancia asintótica (bilateral) &, 029 \\
\hline
\end{tabular}

a. Variable de agrupación: Grupo

Fuente: elaboración propia

Otro dato relevante es el aumento de $60 \%$ a $73,5 \%$ en la aprobación respecto del año anterior, en la asignatura de Psicología del aprendizaje y del desarrollo en la que se realizó la intervención. Si bien estos resultados corresponden a cohortes diferentes de estudiantes, la misma asignatura evidencia mejoras en los índices de eficiencia interna medidos por la universidad.

b. Valoraciones de los participantes en relación con su participación en el programa de tutoría entre iguales

A continuación, se presentan las valoraciones de los participantes organizadas en categorías que emergieron en el proceso de análisis. En la descripción de estos resultados se incluyen figuras que sistematizan conceptos y presentan extractos del discurso de tutores y tutorados. 
Valoración general de la estrategia por parte de los tutores y tutorados

Como se presenta en la figura 1, las valoraciones positivas de los participantes respecto al programa se sustentan en la posibilidad de trabajar de manera personalizada y complementaria los contenidos de la asignatura, lo cual se transforma en un apoyo para el proceso de aprendizaje. En esta instancia, tutor y tutorado tienen una relación cercana en cuanto ambos son estudiantes de la misma carrera de Pedagogía. Los participantes consideran que esta relación propicia la buena comunicación entre ambos, en la que prima la confianza para hacer preguntas, puesto que los estudiantes no se atreven a realizar consultas al profesor frente a toda la clase. Otro aspecto destacado por los futuros maestros es la mediación entre pares como una experiencia de aprendizaje en sí misma, pues modela la idea de la construcción de aprendizaje como un proceso personal y social. Manifiestan que la experiencia es diferente a la que suelen observar en la mayoría de las clases en las cuales participan como estudiantes.

Figura 1

Evaluación de la estrategia por parte de los tutores y tutorados

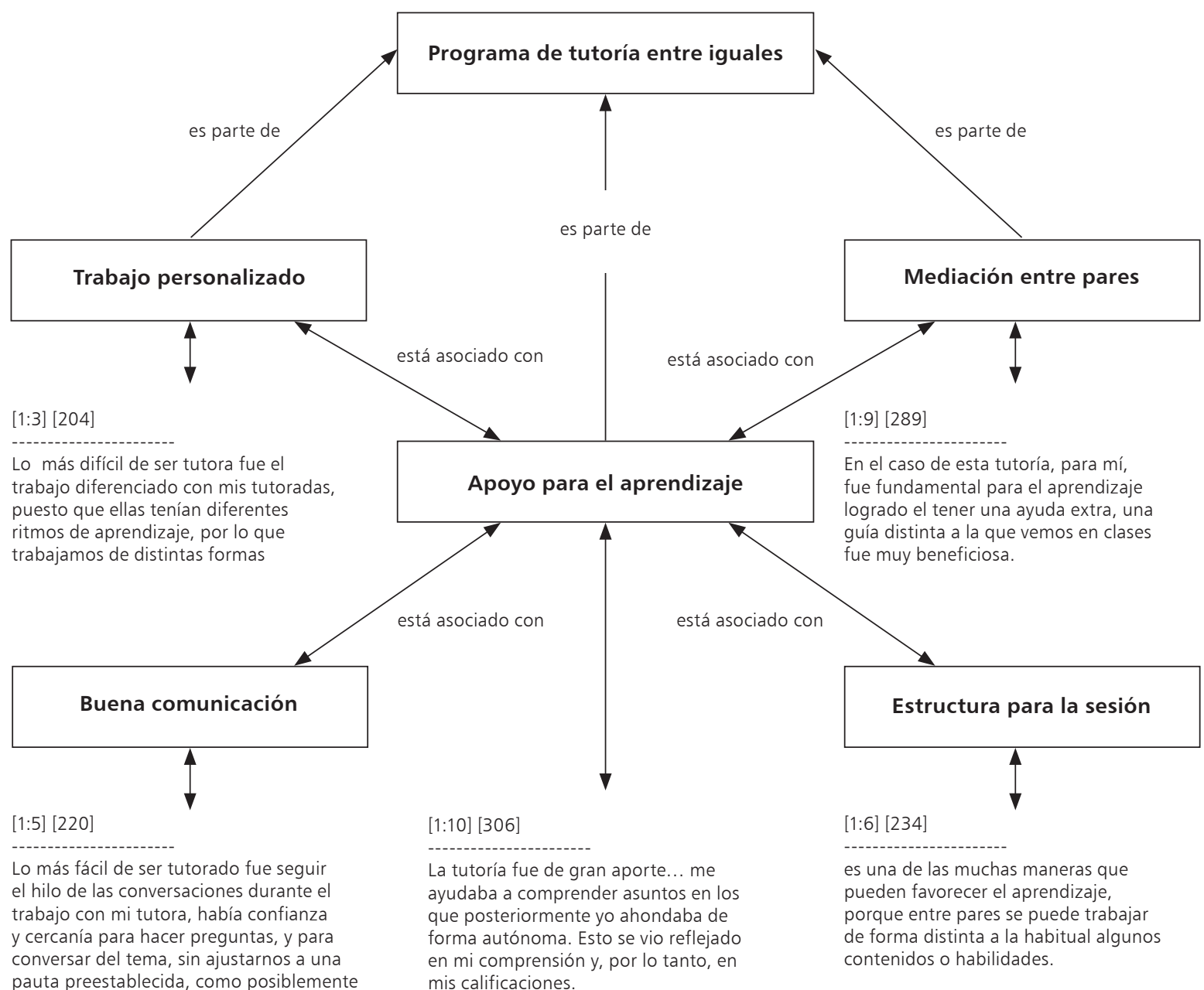

pauta preestablecida, como posiblemente mis calificaciones.

Fuente: elaboración propia 
Al consultar sobre aspectos a mejorar en el programa, los tutores expresan que sus tutorados al inicio veían la sesión de trabajo semanal como un espacio de estudio único y compartido, no complementario a sus labores autónomas como estudiante. Este hecho dificultó la implementación del programa durante el primer mes porque los tutorados no asumían los compromisos acordados con su par. Este hecho cambió a medida que avanzaba el programa, dado que los tutorados se dieron cuenta de que no era una tarea más, sino que resultaba ser un apoyo útil para su aprendizaje.

\section{Reconocimiento de qué se aprende}

Los participantes del programa consideran que la estrategia favorece diferentes tipos de aprendizaje. Como se muestra en la figura 2, desde su perspectiva, los estudiantes visualizan que existe una relación simétrica entre tutor y tutorado, diferente a la que establecen con un profesor de asignatura. Esta relación parece sustentar y fortalecer el proceso de construcción de aprendizajes.

Figura 2

El aprendizaje según tutores y tutorados

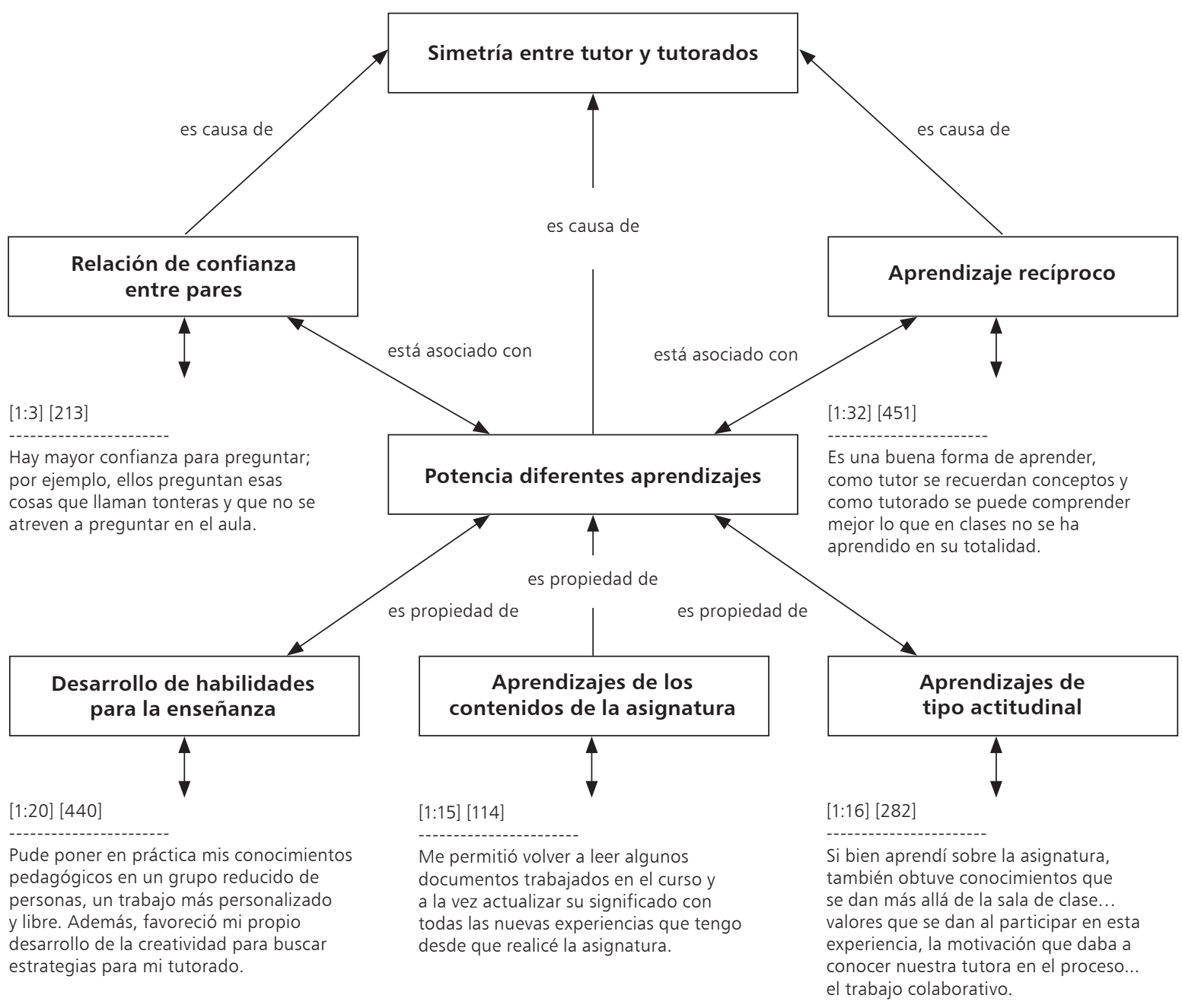


Según las opiniones de los participantes, hay unanimidad en cuanto al aprendizaje recíproco que se logra con la tutoría entre iguales. Tanto tutores como tutorados explican que se han favorecido diferentes tipos de aprendizaje, destacan la profundización en los contenidos de la asignatura de Psicología del aprendizaje, junto con el desarrollo de actitudes positivas como la motivación, responsabilidad y autonomía, además de la posibilidad de aprender a trabajar colaborativamente y a desarrollar habilidades sociales.

Otros aprendizajes que van más allá de los contenidos propios de la asignatura y de la ética profesional son los relacionados con el desarrollo de habilidades para la enseñanza. En específico, los tutores valoran la oportunidad que ofrece el programa al poner en práctica las competencias fundamentales de un profesor. En este sentido, asumir el rol de tutor es complejo porque los desafía a movilizar los recursos y conocimientos pedagógicos con los cuales cuentan para lograr los propósitos de la tutoría entre iguales.

\section{Implicancias de la tutoría entre iguales en la formación de profesores}

A partir del análisis de la información, se develaron implicancias de la estrategia en el proceso formativo, dado que se le considera una estrategia didáctica adecuada para aprender y desarrollar habilidades para la enseñanza; se destaca además que fortalece la vocación docente. Una síntesis de la información se presenta en la figura 3.

Figura 3

Implicancias de la tutoría entre iguales en la formación de profesores

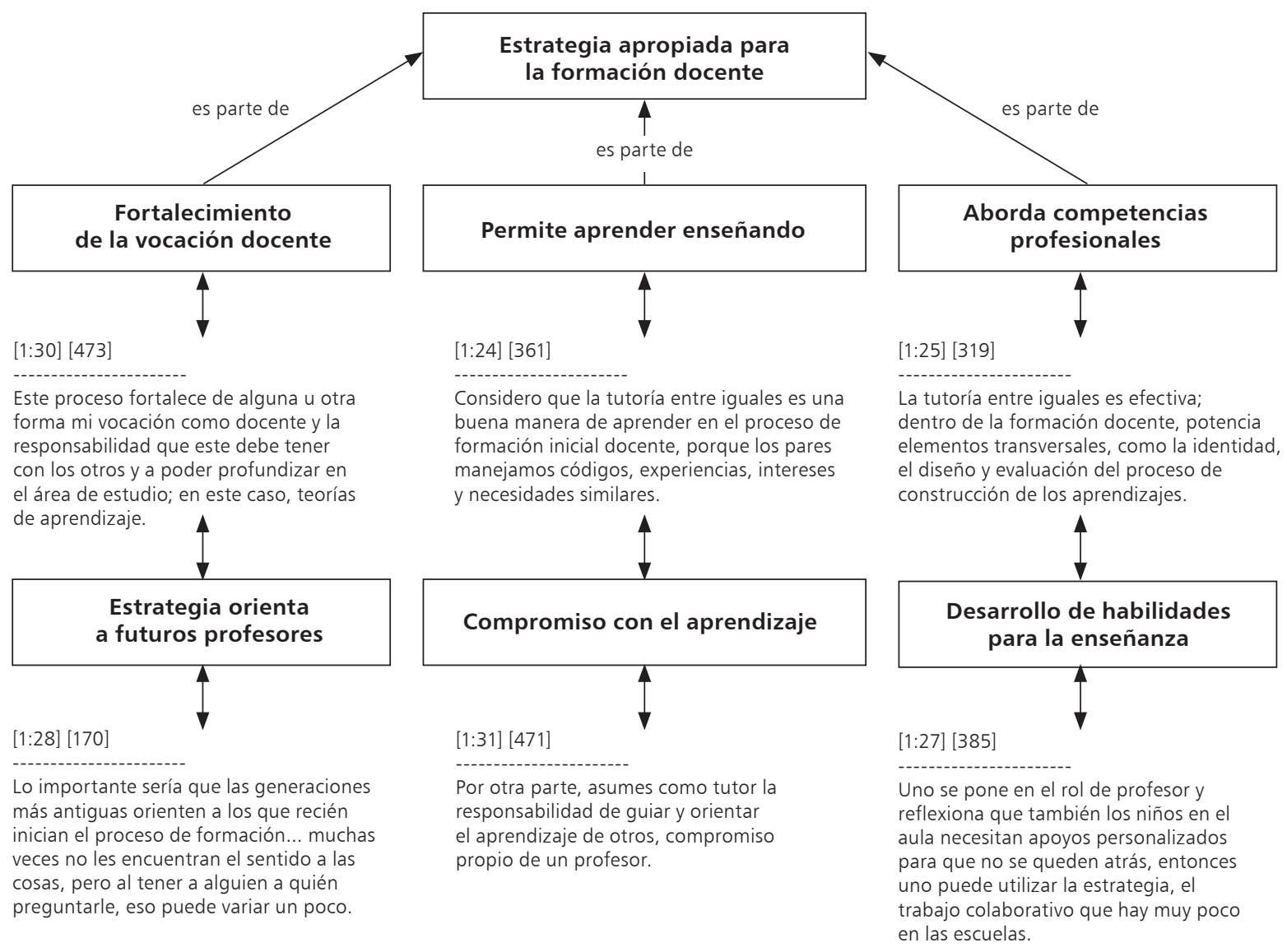

Fuente: elaboración propia 
Los tutores expresan que cuando reflexionan desde el rol de profesores visualizan la forma de poner en práctica esta estrategia de trabajo colaborativo en otros contextos, otras modalidades y grupos de estudiantes, que pueden ser adultos, jóvenes, niños y niñas. De igual manera, la planificación de las sesiones los desafía constantemente a orientar el aprendizaje de sus tutorados, hecho que se concreta en el momento mismo de la mediación que realizan en cada sesión y en el proceso de reflexión que desarrollan en paralelo a todo el proceso de tutoría.

Otro aspecto relevante es la influencia de la estrategia didáctica en el compromiso profesional que están desarrollando los estudiantes como futuros docentes, con el aprendizaje de otros. Tal como lo plantean los participantes, es posible valorar la estrategia de tutoría entre iguales como apropiada para la formación de profesores porque contribuye tanto al logro de los aprendizajes en la carrera de Pedagogía en el caso del tutorado, como al desarrollo de competencias docentes en el caso de quien asume el rol de tutor.

\section{Conclusiones y discusión}

Este estudio ha permitido sistematizar evidencia empírica acerca de un programa de tutoría entre iguales en un contexto universitario y deja de manifiesto el potencial que posee la estrategia como motor de aprendizaje significativo, cuando hay una adecuada estructuración de la interacción entre estudiantes.

Los resultados son consistentes con estudios previos sobre implementaciones de tutoría entre iguales en otros contextos y áreas de estudio (Álvarez \& González, 2005; De Backer, Van Keer \& Valcke, 2016; Duran \& Huerta-Córdova, 2008; González-Fernández, García-Ruiz \& Ramírez-García, 2015; Moliner-Miravet, Sales-Ciges \& Moliner-García, 2014). Así también respecto a investigaciones en el contexto universitario (Duran \& Flores, 2015; Duran, Flores, Mosca \& Santiviago, 2014). Se evidencia que la estrategia de tutoría entre iguales es una forma para nutrir el repertorio de los docentes y enfocarse en el aprendizaje de los estudiantes, la coconstrucción de conocimientos y significados según el enfoque socioconstructivista del aprendizaje.

Es fundamental que las estrategias formativas experimentadas por los futuros maestros sean coherentes con el enfoque de enseñanza que se espera asuman en su ejercicio profesional. En este sentido, el enfoque de la tutoría asume el aprendizaje como un proceso de construcción de conocimiento y no solo de recepción de información. En el programa, asumir el rol de mediador requiere el desarrollo de ayudas ajustadas a las necesidades del tutorado, que construye su aprendizaje desde su nivel de desarrollo real hasta el nivel de desarrollo próximo (Valdebenito, 2012).

En consecuencia, este programa constituye una experiencia formativa más para los futuros profesores de Educación Primaria asumiendo que los maestros enseñarán como han sido formados (Ezquerra-Martínez, De Juanas-Oliva \& Martín del Pozo, 2015). Esta potencialidad de la estrategia se suma a los propósitos más conocidos: favorecer el aprendizaje, fortalecer los procesos de inducción y apoyo a los estudiantes durante su proceso de formación profesional y mejorar los resultados de eficiencia interna en las instituciones de educación superior.

Los participantes del programa expresan una valoración positiva de la experiencia. En este sentido, es relevante reiterar que no solo se brinda apoyo al tutorado, sino que se ofrece otra oportunidad de desarrollo a quien asume el rol de tutor. Desde el paradigma aprender enseñando, es 
posible sustentar y comprender por qué en la tutoría entre iguales, los beneficios son para ambos estudiantes (Duran, Blanch, Corcelles, Flores, Oller, Utset \& Valdebenito, 2011). Estos beneficios contemplan por ejemplo para el tutor, el desarrollo de habilidades y competencias para anticipar y ejercer la docencia, en cuanto implica planificar, enseñar y evaluar los procesos de aprendizaje de otros compañeros o estudiantes; y en el caso del tutorado, un apoyo directo en la construcción de conocimiento, superación de dificultades y desarrollo profesional.

En síntesis, se espera que a partir de los antecedentes aportados por experiencias como esta, los programas de Pedagogía formalicen espacios e incluyan en los itinerarios formativos estructurados para el desarrollo de competencias, estrategias como la tutoría entre iguales, que favorece y diversifica las oportunidades de aprendizaje. Es importante tener en cuenta que el aprendizaje cooperativo y la cooperación en sí misma son un valor social transversal (Echeita Sarrionandia, 2012), por lo cual la estrategia no se justifica solo metodológicamente, sino que en la competencia que moviliza. En este sentido, uno de los desafíos de la formación sería avanzar hacia estructuras que favorezcan la colaboración y la cooperación como una estrategia habitual y consolidada, además del tradicional trabajo individual.

\section{Agradecimientos y aclaraciones}

La investigación se ha desarrollado bajo el patrocinio del proyecto Incremento de Publicaciones Científicas, de la Vicerrectoría de Investigación y Posgrado de la Universidad Católica de Temuco; y de la iniciativa del Fondo Nacional de Desarrollo Científico y Tecnológico Fondecyt 11130216, de la Comisión Nacional de Investigación Científica y Tecnológica, Conicyt.

\section{Sobre las autoras}

Carolina Villagra-Bravo es magíster en Educación con mención Evaluación Educacional, académica de la Universidad Católica de Temuco, UCT, Chile. Docente de pregrado y posgrado.

Vanessa Valdebenito-Zambrano es doctora en Psicología de la Educación, académica e investigadora de la Universidad Católica de Temuco, UCT, Chile. Docente de pregrado, educación continua y posgrado. Miembro del grupo de investigación sobre aprendizaje entre iguales (Grup de Recerca sobre Aprenentatge entre Iguals, GRAI) de la Universidad Autónoma de Barcelona, España. Actualmente, dirige el proyecto Incremento de Publicaciones Científicas, de la Vicerrectoría de Investigación y Posgrado de la Universidad Católica de Temuco, UCT y del Fondo Nacional de Desarrollo Científico y Tecnológico Fondecyt Iniciación 11130216.

\section{Referencias}

Álvarez-Pérez, P. R. \& González-Afonso, M. C. (2005). La tutoría entre iguales y la orientación universitaria. Una experiencia de formación académica y profesional. Educar, 36, 107-128. Disponible en: https://ddd.uab.cat/pub/educar/0211819Xn36/0 211819Xn36p107.pdf

Alzate-Medina, G. M. \& Peña-Borrero, L. B. (2010). La tutoría entre iguales: una modalidad para el desarrollo de la escritura en la educación superior. Universitas Psychologica, 9 (1), 123-138. Disponible en: https://revistas.javeriana.edu.co/index. php/revPsycho/article/view/717

Backer, L. de; Keer, H. van \& Valcke, M. (2016). Eliciting Reciprocal Peer Tutoring Groups' Metacognitive Regulation through Structuring and Problematizing Scaffolds. The Journal of Experimental Education, 84 (4), 804-828. DOI: 10.1080/00220973.2015.1134419. Disponible en: https://www.researchgate.net/publication/ 301236418_Eliciting_Reciprocal_Peer-Tutoring Groups'_Metacognitive_Regulation_Through_ Structuring_and_Problematizing_Scaffolds

Beneitone, P.; Esquetini, C.; González, J.; Maletá, M. M.; Siufi, G. \& Wagenaar, R. (2007). Reflexiones y perspectivas de la Educación Superior en Latinoamérica. Informe Final _Proyecto Tuning-, América Latina, 2004-2007. Bilbao, Groningen: Universidad de Deusto, Universidad de Groningen. Disponible en: http://tuningacademy.org/ wp-content/uploads/2014/02/TuningLAIII_Fi nal-Report_SP.pdf

Blanch-Gelabert, S.; Corcelles-Seuba, M.; Duran-Gisbert, D.; Dekhinet, R. \& Topping, K. (2014). La escritura y corrección de textos en una tutoría entre iguales, recíproca y virtual, para la mejora en inglés y español. Revista de Educación, 363, 309-333. DOI: 10.4438/1988-592X-RE-2012-363-190. Disponible en: http://www.educacionyfp.gob.es/re vista-de-educacion/numeros-revista-educacion/ numeros-anteriores/2014/363.html

Blanch, S.; Duran, D.; Dekhinet, R. \& Topping, K. (2010). Una experiencia de tutoría entre iguales virtual para el aprendizaje del castellano y el inglés. Textos de Didáctica de la Lengua y la Literatura, 53, 89-101. Disponible en: http://grupsderecerca. uab.cat/grai/sites/grupsderecerca.uab.cat.grai/ files/tutoriaentreigualescastellaingles.pdf

Blanch, S.; Duran, D.; Valdebenito, V. \& Flores, M. (2013). The Effects and Characteristics of Family Involvement on a Peer Tutoring Programme to Improve the Reading Comprehension Competence. European Journal of Psychology of Education, 28 (1), 101-119. 
Cortese, C. G. (2005). Learning through Teaching. Management Learning, 36 (1), 87-115. https://doi.org/10.1177/1350507605049905.

Delors, J. (1996). Learning the Treasure within. Report to UNESCO of the International Commission on Education for the Twenty-First Century. Paris: United Nations Educational, Scientific and Cultural Organization, UNESCO. Disponible en: https://unesdoc.unesco.org/ ark:/48223/pf0000109590

Duran, D. (2014). Aprenseñar. Evidencias e implicaciones educativas de aprender enseñando. Madrid: Narcea.

Duran, D. (coord.); Blanch, S.; Corcelles, M.; Flores, M.; Oller, M.; Utset, M. \& Valdebenito, V. (2011). Leemos en pareja. Tutoría entre iguales para la competencia lectora. Barcelona: Horsori.

Duran, D. \& Flores, M. (2015). Prácticas de tutoría entre iguales en universidades del Estado español y de Iberoamérica. Revista Iberoamericana sobre Calidad, Eficacia y Cambio en Educación, REICE, 13 (1), 5-17. Disponible en: https://revistas.uam.es/index.php/reice/article/ view/2796/3011

Duran, D.; Flores, M.; Mosca, A. \& Santiviago, C. (2014). Tutoría entre iguales, del concepto a la práctica en las diferentes etapas educativas. InterCambios, 2 (1), 31-39. Disponible en: http://grupsderecerca.uab. cat/grai/sites/grupsderecerca.uab.cat.grai/files/art3_duran.pdf

Duran, D. \& Huerta-Córdova, V. (2008). Una experiencia de tutoría entre iguales en la Universidad mexicana de Oaxaca. Revista Iberoamericana de Educación, 48 (1), 1-12. Disponible en: https://rieoei.org/RIE/ article/view/2262

Duran, D. \& Monereo, C. (2005). Styles and Sequences of Cooperative Interaction in Fixed and Reciprocal Peer Tutoring. Learning and Instruction, 15 (3), 179-199. DOI: 10.1016/j.learninstruc.2005.04.002. Disponible en: https://www.researchgate.net/ profile/David_Duran5/publication/222122432_Styles_and_sequen ce_of_cooperative_interaction_in_fixed_and_reciprocal_peer_tu toring/links/5a19ae52aca272df080d7e3d/Styles-and-sequenceof-cooperative-interaction-in-fixed-and-reciprocal-peer-tutoring. pdf?origin=publication_detail

Duran, D. \& Sánchez-Chacón, G. (2012). Ritmos en dos: una experiencia basada en la tutoría entre iguales para la mejora de la fluidez y comprensión de lectura rítmica musical. Eufonía. Didáctica de la Música, 56, 99-106. Disponible en: http://grupsderecerca.uab.cat/grai/sites/ grupsderecerca.uab.cat.grai/files/ritmos.pdf

Duran, D. \& Utset, M. (2014). Red Leemos en pareja: un modelo de formación basado en el aprendizaje entre iguales para la sostenibilidad de la innovación educativa. Cultura y Educación, 26 (2), 377-384. https://doi.org/10.1080/11356405.2014.935109

Duran, D. \& Vidal-Iglesias, V. (2004). Tutoría entre iguales: de la teoría a la práctica. Un método de aprendizaje cooperativo para la diversidad en secundaria. Barcelona: Graó.

Echeita-Sarrionandia, G. (2012). El aprendizaje cooperativo al servicio de una educación de calidad. Cooperar para aprender y aprender para cooperar. En J. C. Torrego García \& A. Negro Moncayo (coords.) Aprendizaje cooperativo en las aulas. Fundamentos y recursos para su implantación, 21-46. Madrid: Alianza Editorial.

Ezquerra-Martínez, Á.; Juanas-Oliva, Á. de \& Martín del Pozo, R. (2015). Estudio sobre las actividades llevadas a cabo en la práctica docente universitaria para la formación inicial del profesorado de primaria y secundaria. Profesorado, Revista de Currículum y Formación del 
Profesorado, 19 (1), 330-345. Disponible en: http://www.redalyc.org/ pdf/567/56738729020.pdf

Flores, M. \& Duran, D. (2016). Influence of a Catalan Peer Tutoring Programme on Reading Comprehension and Self-Concept as a Reader. Journal of Research in Reading, 39 (3), 330-346. DOI:10.1111/14679817.12044. Disponible en: http://grupsderecerca.uab.cat/grai/sites/ grupsderecerca.uab.cat.grai/files/Influence $\% 20$ of\%20a\%20cata lan...\%20Journal\%20of\%20Research\%20in\%20Reading.pdf

Fuchs, L.; Fuchs, D.; Hamlett, C.; Phillips, N.; Karns, K. \& Dutka, S. (1997). Enhancing Students' Helping Behavior during Peer-Mediated Instruction with Conceptual Mathematical Explanations. Elementary School Journal, 97 (3), 223-249.

Garagorri-Yarza, X. (2007). Currículo basado en competencias: aproximación al estado de la cuestión. Aula de Innovación Educativa, 161, 4755. Disponible en: http://recursos.cepindalo.es/pluginfile.php/7384/ mod_resource/content/1/OPINION_xabier_garagorri.pdf

Gillies, R. M. (2007). Cooperative Learning. Integrating Theory and Practice. Los Angeles, California: Sage.

González-Fernández, N.; García-Ruiz, R. \& Ramírez-García, A. (2015). Aprendizaje cooperativo y tutoría entre iguales en entornos virtuales universitarios. Estudios Pedagógicos, 41 (1), 111-124. Disponible en: http://www.scielo.cl/pdf/estped/v41n1/art07.pdf

Good, T. \& Brophy, J. (2000). Looking in Classrooms. New York: Longman. Mastropieri, M. A.; Scruggs, T. E.; Norland, J. J.; Berkeley, S.; McDuffie, K., Halloran Tornquist, E. \& Connors, N. (2006). Differentiated Curriculum Enhancement in Middle-School Science: Effects on Classroom and High-Stakes Test. The Journal of Special Education, 40 (3), 130137. DOI: $10.1177 / 00224669060400030101$. Disponible en: https:// pdfs.semanticscholar.org/a5fb/407d221921ed2499494ee387cb7105 5a7ac5.pdf

Mastropieri, M. A.; Scruggs, T. E.; Spencer, V. \& Fontana, J. (2003). Promoting Success in High School World History: Peer Tutoring versus Guided Notes. Learning Disabilities, Research and Practice, 18 (1), 52-65. https://doi.org/10.1111/1540-5826.00057

Moliner-Miravet, L.; Flores-Coll, M. \& Duran-Gisbert, D. (2011). Efectos sobre la mejora de las competencias lingüísticas y la autoimagen lectora a través de un programa de tutoría entre iguales. Revista de Investigación en Educación, 9 (2), 209-222. Disponible en: http:// grupsderecerca.uab.cat/grai/sites/grupsderecerca.uab.cat.grai/files/ efectosmejoraeuskadi.pdf

Moliner-Miravet, L.; Sales-Ciges, A. \& Moliner-García, O. (2014). An Experience of Reciprocal Peer Tutoring at the University. Procedia and Behavioral Sciences, 116, 2809-2812. https://doi.org/10.1016/ j.sbspro.2014.01.661. Disponible en: http://ac.els-cdn.com/S18770 42814006788/1-s2.0-S1877042814006788-main.pdf?

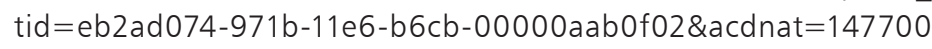
5864_cf3e8156232ad084d74974bc8b52ae7c

Monereo-Font, C. (2001). La enseñanza estratégica: enseñar para la autonomía. En C. Monereo Font (coord.), A. Badia Garganté, M. V. Baixeras Fecé, E. Boadas Mir, M. Castelló Badia, I. Guevara Casanova, E. Miquel Bertrán, M. Monte Aneas, E. M. Sebastiáni Obrador. Ser estratégico y autonomo aprendiendo. Unidades didácticas de enseñanza estratégica para la ESO [Educación Secundaria Obligatoria], 11 27. Barcelona: Graó. 
Mortweet, S. L.; Utley, C. A.; Walker, D.; Dawson, H. L; Delquadri, J. C.; Reddy, S. S.; Greenwood, C. R.; Hamilton, S. \& Ledford, D. (1999). Classwide Peer Tutoring: Teaching Students with Mild Mental Retardation in Inclusive Classrooms. Exceptional Children, 65 (4), 524-536.

Mosca, A. \& Santiviago, C. (2012). Fundamentos conceptuales de las tutorías entre pares. La experiencia de la Universidad de la República. Montevideo, Uruguay: Universidad de la República. Disponible en: https://do cplayer.es/84404-Fundamentos-conceptuales-de-las-tutorias-entrepares-la-experiencia-de-la-universidad-de-la-republica.html

Organización de Estados Iberoamericanos para la Educación, la Ciencia y la Cultura, OEI (2010). Metas Educativas 2021. La educación que queremos para la generación de los bicentenarios. Madrid: OEl. Disponible en: https://www.oei.es/historico/metas2021/libro.htm

Organización para la Cooperación y el Desarrollo Económicos, OCDE (2005). La definición y selección de competencias clave. Resumen ejecutivo. Disponible en: http://deseco.ch/bfs/deseco/en/index/03/02. parsys.78532.downloadList.94248.DownloadFile.tmp/2005.dscexe cutivesummary.sp.pdf

Parrilla-Latas, Á. (2002). Acerca del origen y sentido de la Educación Inclusiva. Revista de Educación, 327, 11-29. Disponible en: http:// www.mecd.gob.es/dctm/revista-de-educacion/articulos327/ re3270210520.pdf?documentld=0901e72b81259a76

Pérez-Gómez, Á. I. (2007). Las competencias básicas y el currículo: orientaciones generales. Gobierno de Cantabria: Consejería de Educación. Disponible en: https://www.educantabria.es/docs/info_institucional/ publicaciones/2007/Cuadernos_Educacion_2.PDF

Robinson, D. R.; Schofield, J. W. \& Steers-Wentzell, K. L. (2005). Peer and Cross-Age Tutoring in Math: Outcomes and Their Design Implications. Educational Psychology Review, 17 (4), 327-362.

Roscoe, R. D. \& Chi, M. T. H. (2007). Understanding Tutor Learning: Knowledge-Building and Knowledge-Telling in Peer Tutors' Explanations and Questions. Review of Educational Research, 77 (4), 534574. https://doi.org/10.3102/0034654307309920

Ryan, J.; Reid, R. \& Epstein, M. H. (2004). Peer-Mediated Intervention Studies on Academic Achievement for Students with EBD [Emotional and Behavior Disorders]: A Review. Remedial and Special Education, 25 (6), 330-341. DOI: 10.1177/07419325040250060101. Disponible en: https://www.researchgate.net/publication/249835090_PeerMediated_Intervention_Studies_on_Academic_Achievement_for_ Students_with_EBD_A_Review

Sabariego, M. \& Bisquerra, R. (2004). Fundamentos metodológicos de la investigación educativa. En R. Bisquerra (coord.). Metodología de la investigación educativa, 20-49. Madrid: La Muralla.

Stake, R. (2005). Investigación con estudio de caso. Madrid: Morata.

Tapia-Gutiérrez, C. P. (2012). Desarrollo de un modelo representacional y un instrumento para la evaluación de las habilidades sociales en contextos universitarios. Tesis doctoral publicada. Universidad de Extremadura.

Thurston, A.; Keere, K. van de; Topping, K. J.; Kosack, W.; Gatt, S.; Marchal, J.; Mestdagh, N.; Schmeinck, D.; Sidor, W. \& Donnert, K. (2007). Aprendizaje entre iguales en Ciencias Naturales de Educación Primaria: Perspectivas teóricas y sus implicaciones para la práctica en el aula. Revista Electrónica de Investigación Psicoeducativa [Electronic Journal of Research in Educational Psychology], 5 (3), 477-496. Disponible en: https://www.redalyc.org/articulo.oa?id=293121946002 
Topping, K. J. (1988). The Peer Tutoring Handbook: Promoting Co-operative Learning. London: Croom Helm.

Topping, K. J. (1996). Effective Peer Tutoring in Further and Higher Education (SEDA Paper 95). Birmingham: Staff and Educational Development Association, SEDA.

Topping, K. J. (2000). Tutoring by Peers, Family, and Volunteers. Geneva: UNESCO

Topping, K.; Duran, D. \& Keer, H. van (2015). Using Peer Tutoring to Improve Reading Skills. New York: Routledge.

Universidad Católica de Temuco, UCT (2007). Modelo educativo UC Temuco. Principios y lineamientos. Temuco: Universidad Católica de Temuco. Disponible en: http://www.uct.cl/archivos/modeloeducativo.pdf

Valdebenito, V. (2012). Desarrollo de la competencia lectora, comprensión y fluidez, a través de un programa de tutoría entre iguales, como metodología para la inclusión (tesis doctoral). Universidad Autónoma de Barcelona, Barcelona, España. Disponible en: https://ddd.uab.cat/ pub/tesis/2011/hdl_10803_96309/vhvz1de1.pdf

Valdebenito, V. \& Duran, D. (2015). Formas de interacción implicadas en la promoción de estrategias de comprensión lectora a través de un programa de tutoría entre iguales. Revista Latinoamericana de Psicología, 47 (2), 75-85. Disponible en: http://www.elsevier.es/es-revistarevista-latinoamericana-psicologia-205-pdf-S0120053415000023

Vezub, L. F. (2007). La formación y el desarrollo profesional docente frente a los nuevos desafíos de la escolaridad. Profesorado, Revista de Currículum y Formación del Profesorado, 11 (1), 1-23. Disponible en: https://www.ugr.es/ recfpro/rev111ART2.pdf

Zabala, A. \& Arnau, L. (2007). 11 ideas clave. Cómo aprender y enseñar competencias. Barcelona: Graó. 